J. Korean Math. Soc. 46 (2009), No. 5, pp. 949-966

DOI 10.4134/JKMS.2009.46.5.949

\title{
ON COMPLEX FINSLER SPACES WITH RANDERS METRIC
}

\author{
Nicoleta Aldea And Gheorghe Munteanu
}

\begin{abstract}
In this paper we introduce in study a new class of complex Finsler spaces, namely the complex Randers spaces, for which the fundamental metric tensor and the Chern-Finsler connection are determined. A special approach is devoted to Kähler-Randers metrics. Using the length arc parametrization for the extremal curves of the Euler-Lagrange equations we obtain a complex nonlinear connections of Lorentz type in a complex Randers space.
\end{abstract}

\section{Introduction}

The real Randers metrics were first introduced by G. Randers in the context of general relativity and they were applied to the theory of the electron microscope by R. Ingarden [11]. The importance of real Randers spaces is also pointed out in [6] and the obtained results are remarkable. Recently, it was shown that the real Randers metrics are solutions to Zermelo's navigation problem ([8]) and the classification of real Randers metrics of constant flag curvature was finally completed $([6,8,21,13])$.

As compared to the real case, in complex Finsler geometry there are not so many known classes of complex Finsler metrics. Besides the significant Kobayashi and Caratheodory metrics (see [1]), which quickened the study of such Finsler geometry, we know two rather trivial classes of complex Finsler metrics: the complex Finsler metrics which come from Hermitian metrics on the base manifold (the purely Hermitian metrics in [15]), and the locally Minkowski complex metrics. Therefore, any new class of complex Finsler spaces with some meaning in both theory and applications is welcome.

In the present paper, we have three goals. The first goal is to introduce the complex Randers metrics, i.e., complex metrics constructed from just two pieces of familiar data: a purely Hermitian metric and a differential $(1,0)$-form, both globally defined on an underlying complex manifold. We show that the complex Randers metric thus built is a complex Finsler metric (Theorem 2.1). We

Received November 13, 2007; Revised February 19, 2008.

2000 Mathematics Subject Classification. 53B40, 53C56.

Key words and phrases. complex Finsler spaces, Randers spaces. 
determine the fundamental metric tensor of a complex Randers space (Proposition 2.1), its inverse and determinant, (Proposition 2.3). Moreover, a complex Randers metric also produces another invertible $d$-tensor, (Proposition 2.4). By deformation of some purely Hermitian metrics we obtain examples of complex Randers metric.

The second goal is to find the conditions such that a complex Randers space is weakly Kähler or moreover, strongly Kähler. A special attention is devoted to a class of complex Randers metrics with an additional assumption. We find a necessary and sufficient condition that such a complex Randers metric should be weakly Kähler (Proposition 3.1) and also some outcomes about strongly Kähler-Randers metrics, (Propositions 3.2, 3.3). At the end of Section 3 our results are applied to some examples, better illustrating the interest for this work.

Our third goal is the study of the variational problem for the Lagrangian of a complex Randers space. This approach is somewhat different than the classical one known in complex Finsler spaces $([17,15])$. As in the real case, [14], using the canonical parametrization for the extremal curves of the EulerLagrange equations, we obtain the Lorentz equations of a complex Randers space and from these a complex nonlinear connection of Lorentz type is deduced (Proposition 4.3).

The study that we made in this paper often contains computational arguments (cf. real case), some of which are presented in detail here while in other cases we resumed to only sketching the essential ideas. In our view, the effort to decipher these computations is rewarded by the fact that this paper offers a new interesting class of complex Finsler spaces.

A first draft of this paper was presented at Nat. Sem. on Finsler, Lagrange and Hamilton spaces, Braşov, Sept., 2006. A short outline of these results will be found in the Seminar's volume and some ideas were already cited by B. Chen and Y. Shen in a recent work $([9])$. The present paper completes and clarifies better the idea of complex Randers space. Moreover, we hope that this class of metrics will offer a geometrical model, especially for quantum physics theories.

In the following, let us briefly set the basic notions which are needed; for more information see $[1,15]$.

Let $M$ be a complex manifold, $\operatorname{dim}_{C} M=n$. The complexified of the real tangent bundle $T_{C} M$ splits into the sum of holomorphic tangent bundle $T^{\prime} M$ and its conjugate $T^{\prime \prime} M$. The bundle $T^{\prime} M$ is in its turn a complex manifold, the local coordinates in a chart will be denoted by $u=\left(z^{k}, \eta^{k}\right)$ and these are changed by the rules: $z^{\prime k}=z^{\prime k}(z), \eta^{\prime k}=\frac{\partial z^{\prime k}}{\partial z^{j}} \eta^{j}$. The complexified tangent bundle of $T^{\prime} M$ is decomposed as $T_{C}\left(T^{\prime} M\right)=T^{\prime}\left(T^{\prime} M\right) \oplus T^{\prime \prime}\left(T^{\prime} M\right)$. A natural local frame for $T_{u}^{\prime}\left(T^{\prime} M\right)$ is $\left\{\frac{\partial}{\partial z^{k}}, \frac{\partial}{\partial \eta^{k}}\right\}$, which have changes by the rules obtained with Jacobi matrix of above transformations. Note that the change rule of $\frac{\partial}{\partial z^{k}}$ contains the second order partial derivatives. 
Let $V\left(T^{\prime} M\right)=\operatorname{ker} \pi_{*} \subset T^{\prime}\left(T^{\prime} M\right)$ be the vertical bundle, spanned locally by $\left\{\frac{\partial}{\partial \eta^{k}}\right\}$. A complex nonlinear connection, briefly (c.n.c.), determines a supplementary complex subbundle to $V\left(T^{\prime} M\right)$ in $T^{\prime}\left(T^{\prime} M\right)$, i.e., $T^{\prime}\left(T^{\prime} M\right)=$ $H\left(T^{\prime} M\right) \oplus V\left(T^{\prime} M\right)$. It determines an adapted frame $\left\{\frac{\delta}{\delta z^{k}}=\frac{\partial}{\partial z^{k}}-N_{k}^{j} \frac{\partial}{\partial \eta^{j}}\right\}$, where $N_{k}^{j}(z, \eta)$ are the coefficients of the (c.n.c.), ([1], [2], [15]).

A continuous function $F: T^{\prime} M \rightarrow \mathbb{R}^{+}$is called complex Finsler metric on $M$ if it satisfies the conditions:

i) $L:=F^{2}$ is smooth on $\overline{T^{\prime} M}:=T^{\prime} M \backslash\{0\}$;

ii) $F(z, \eta) \geq 0$, the equality holds if and only if $\eta=0$;

iii) $F(z, \lambda \eta)=|\lambda| F(z, \eta)$ for $\forall \lambda \in \mathbb{C}$;

iv) the Hermitian matrix $\left(g_{i \bar{j}}(z, \eta)\right)$, with $g_{i \bar{j}}=\frac{\partial^{2} L}{\partial \eta^{i} \partial \bar{\eta}^{j}}$, called the fundamental metric tensor, is positive definite.

The pair $(M, F)$ is called a complex Finsler space. The iv)-th assumption involves the strongly pseudoconvexity of the Finsler metric $F$ on complex indicatrix $I_{F, z}=\left\{\eta \in T_{z}^{\prime} M \mid F(z, \eta)<1\right\}$.

Further, in a complex Finsler space a Hermitian connection of $(1,0)$-type has a special meaning, named in [1] the Chern-Finsler connection. In notations from [15] it is $D \Gamma N=\left(L_{j k}^{i}, 0, C_{j k}^{i}, 0\right)$, where

$$
\stackrel{C F}{N_{j}^{i}}=g^{\bar{m} i} \frac{\partial g_{l \bar{m}}}{\partial z^{j}} \eta^{l} ; \quad L_{j k}^{i}=g^{\bar{m} i} \frac{\delta g_{j \bar{m}}}{\delta z^{k}}=\frac{\partial N_{k}^{i}}{\partial \eta^{j}} ; \quad C_{j k}^{i}=g^{\bar{m} i} \frac{\partial g_{j \bar{m}}}{\partial \eta^{k}}
$$

Now, let us recall that in [1]'s terminology, the complex Finsler space $(M, F)$ is strongly Kähler if and only if $L_{j k}^{i}-L_{k j}^{i}=0$, Kähler if and only if $\left(L_{j k}^{i}-\right.$ $\left.L_{k j}^{i}\right) \eta^{j}=0$ and weakly Kähler if and only if $g_{i \bar{l}}\left(L_{j k}^{i}-L_{k j}^{i}\right) \eta^{j} \bar{\eta}^{l}=0$. In the particular case of purely Hermitian metrics, that is $g_{i \bar{j}}=g_{i \bar{j}}(z)$, those three nuances of Kähler coincide ([18]).

For the vertical section $\mathcal{L}=\eta^{k} \dot{\partial}_{k}$, with $\dot{\partial}_{k}:=\frac{\dot{\partial}}{\partial \eta^{k}}$, called the Liouville complex field (or the vertical radial vector field in [1]), we consider its horizontal lift $\chi:=\eta^{k} \delta_{k},\left(\delta_{k}:=\frac{\delta}{\delta z^{k}}\right)$.

According to $[1$, p. 108] and [15, p. 81], the holomorphic curvature of the complex Finsler space $(M, F)$ in direction $\eta$ is

$$
\mathcal{K}_{F}(z, \eta)=\frac{2}{L^{2}} \mathbf{G}(\mathbf{R}(\chi, \bar{\chi}) \chi, \bar{\chi})
$$

and locally it has the following expression (see [3])

$$
\mathcal{K}_{F}(z, \eta)=\frac{2}{L^{2}} R_{\bar{j} k} \bar{\eta}^{j} \eta^{k}, \text { where } R_{\bar{j} k}=-g_{l \bar{j}} \delta_{\bar{h}} \stackrel{C F}{\left(N_{k}^{l}\right)} \bar{\eta}^{h}
$$

More informations can be found in $[1,2,3,15,18]$. 


\section{Complex Randers spaces}

Following the ideas from real case, $[6,8,21,13]$, we shall introduce a new class of complex Finsler metrics. We consider $z \in M$ and $\eta \in T_{z}^{\prime} M, \eta=\eta^{i} \frac{\partial}{\partial z^{i}}$. On $M$ let

- $a:=a_{i \bar{j}}(z) d z^{i} \otimes d \bar{z}^{j}$ be a purely Hermitian positive metric and

- $b=b_{i}(z) d z^{i}$ be a differential $(1,0)$-form.

By these objects we define the function $F$ on $T^{\prime} M$

$$
F(z, \eta):=\alpha(z, \eta)+|\beta(z, \eta)|
$$

where

$$
\begin{aligned}
\alpha(z, \eta) & :=\sqrt{a_{i \bar{j}}(z) \eta^{i} \bar{\eta}^{j}} \\
|\beta(z, \eta)| & =\sqrt{\beta(z, \eta) \overline{\beta(z, \eta)}} \text { with } \beta(z, \eta)=b_{i}(z) \eta^{i} .
\end{aligned}
$$

By analogy with the real case, we call the function from (2.1) the complex Randers metric and the pair $(M, \alpha+|\beta|)$ a complex Randers space. Such a metric is only quoted as an example of complex Finsler metric in [10, 20].

Our goal in the sequel is to find the circumstances in which the function (2.1) is a complex Finsler metric. Some remarks are immediate. Due to the presence of $|\beta|$, the complex Randers metric $F:=\alpha+|\beta|$ is positive and smooth on $T^{\prime} M \backslash\{0\}$. The complex Randers metric is purely Hermitian if and only if $\beta$ vanishes identically.

Obviously, the function $L:=F^{2}=(\alpha+|\beta|)^{2}$ depends on $z$ and $\eta$ by means of the real valued functions $\alpha:=\alpha(z, \eta)$ and $|\beta|:=|\beta(z, \eta)|$. Moreover $\alpha$ and $\beta$ are homogeneous with respect to $\eta$, i.e., $\alpha(z, \lambda \eta)=|\lambda| \alpha(z, \eta), \beta(z, \lambda \eta)=\lambda \beta(z, \eta)$ for any $\lambda \in \mathbb{C}$, thus $L(z, \lambda \eta)=\lambda \bar{\lambda} L(z, \eta)$ for any $\lambda \in \mathbb{C}$, and so the homogeneity property implies

$$
\begin{gathered}
\frac{\partial \alpha}{\partial \eta^{i}} \eta^{i}=\frac{1}{2} \alpha ; \quad \frac{\partial|\beta|}{\partial \eta^{i}} \eta^{i}=\frac{1}{2}|\beta| ; \quad L_{\alpha}=L_{|\beta|}=2 F ; \alpha L_{\alpha}+|\beta| L_{|\beta|}=2 L ; \\
\alpha L_{\alpha \alpha}+|\beta| L_{\alpha|\beta|}=L_{\alpha} ; \alpha L_{\alpha|\beta|}+|\beta| L_{|\beta||\beta|}=L_{|\beta|} ; \\
\alpha^{2} L_{\alpha \alpha}+2 \alpha|\beta| L_{\alpha|\beta|}+|\beta|^{2} L_{|\beta||\beta|}=2 L,
\end{gathered}
$$

where $L_{\alpha}:=\frac{\partial L}{\partial \alpha}, L_{|\beta|}:=\frac{\partial L}{\partial|\beta|}, L_{\alpha \alpha}:=\frac{\partial^{2} L}{\partial \alpha^{2}}$, etc.

The main issue that needs to be checked is the strongly pseudoconvexity of the complex Randers function. First we shall determine the fundamental tensor of the complex Randers space $(M, \alpha+|\beta|)$, i.e., $g_{i \bar{j}}=\partial^{2}(\alpha+|\beta|)^{2} / \partial \eta^{i} \partial \bar{\eta}^{j}$. For this, let us consider the settings

$$
\begin{aligned}
\frac{\partial \alpha}{\partial \eta^{i}} & =\frac{1}{2 \alpha} l_{i} ; \frac{\partial|\beta|}{\partial \eta^{i}}=\frac{\bar{\beta}}{2|\beta|} b_{i} ; \\
b^{i} & :=a^{\bar{j} i} b_{\bar{j}} ; \quad\|b\|^{2}:=a^{\bar{j} i} b_{i} b_{\bar{j}} ; \gamma:=L+\alpha^{2}\left(\|b\|^{2}-1\right) ; \\
\eta_{i} & :=\frac{\partial L}{\partial \eta^{i}}=L_{\alpha} \frac{\partial \alpha}{\partial \eta^{i}}+L_{|\beta|} \frac{\partial|\beta|}{\partial \eta^{i}}=\frac{F}{\alpha} l_{i}+\frac{F \bar{\beta}}{|\beta|} b_{i},
\end{aligned}
$$


where $l_{i}:=a_{i \bar{j}} \bar{\eta}^{j}$ and $\left(a^{\bar{j} i}\right)$ is the Hermitian inverse of $\left(a_{i \bar{j}}\right)$ matrix.

Proposition 2.1. The fundamental metric tensor of the complex Randers metric $F:=\alpha+|\beta|$ is given by

$$
g_{i \bar{j}}=\frac{F}{\alpha} h_{i \bar{j}}+\frac{F}{2|\beta|} b_{i} b_{\bar{j}}+\frac{1}{2 L} \eta_{i} \eta_{\bar{j}}
$$

where $h_{i \bar{j}}:=a_{i \bar{j}}-\frac{1}{2 \alpha^{2}} l_{i} l_{\bar{j}}$.

Proof. Indeed, from $g_{i \bar{j}}=\frac{\partial \eta_{i}}{\partial \bar{\eta}^{j}}=L_{\alpha \alpha} \frac{\partial \alpha}{\partial \bar{\eta}^{j}} \frac{\partial \alpha}{\partial \eta^{i}}+L_{\alpha|\beta|}\left(\frac{\partial|\beta|}{\partial \bar{\eta}^{j}} \frac{\partial \alpha}{\partial \eta^{i}}+\frac{\partial \alpha}{\partial \bar{\eta}^{j}} \frac{\partial|\beta|}{\partial \eta^{i}}\right)+$ $L_{|\beta||\beta|} \frac{\partial|\beta|}{\partial \bar{\eta}^{j}} \frac{\partial|\beta|}{\partial \eta^{i}}+L_{\alpha} \frac{\partial^{2} \alpha}{\partial \eta^{i} \partial \bar{\eta}^{j}}+L_{|\beta|} \frac{\partial^{2}|\beta|}{\partial \eta^{i} \partial \bar{\eta}^{j}}$ and (2.3), we deduce (2.4).

The next goal is to find the formulas for the determinant and the inverse of the fundamental tensor $g_{i \bar{j}}$. The solution is obtained by adapting Proposition 11.2.1, p. 287 from [6] for an arbitrary non-singular Hermitian matrix $\left(Q_{i \bar{j}}\right)$. The result is,

\section{Proposition 2.2. Suppose:}

- $\left(Q_{i \bar{j}}\right)$ is a non-singular $n \times n$ complex matrix with inverse $\left(Q^{\bar{j} i}\right)$;

- $C_{i}$ and $C_{\bar{\imath}}:=\overline{C_{i}}, i=1, \ldots, n$, are complex numbers;

- $C^{i}:=Q^{\bar{j} i} C_{\bar{j}}$ and its conjugates; $C^{2}:=C^{i} C_{i}=\bar{C}^{i} C_{\bar{\imath}} ; H_{i \bar{j}}:=Q_{i \bar{j}} \pm C_{i} C_{\bar{j}}$.

Then

i) $\operatorname{det}\left(H_{i \bar{j}}\right)=\left(1 \pm C^{2}\right) \operatorname{det}\left(Q_{i \bar{j}}\right)$;

ii) Whenever $1 \pm C^{2} \neq 0$, the matrix $\left(H_{i \bar{j}}\right)$ is invertible and in this case its inverse is $H^{\bar{j} i}=Q^{\bar{j} i} \mp \frac{1}{1 \pm C^{2}} C^{i} C^{\bar{j}}$.

Proposition 2.3. For the complex Randers metric $F:=\alpha+|\beta|$ we have

i) $g^{\bar{j} i}=\frac{\alpha}{F} a^{\bar{j} i}+\frac{|\beta|\left(\alpha|| b||^{2}+|\beta|\right)}{L \gamma} \eta^{i} \bar{\eta}^{j}-\frac{\alpha^{3}}{F \gamma} b^{i} \bar{b}^{j}-\frac{\alpha}{F \gamma}\left(\bar{\beta} \eta^{i} \bar{b}^{j}+\beta b^{i} \bar{\eta}^{j}\right)$;

ii) $\operatorname{det}\left(g_{i \bar{j}}\right)=\left(\frac{F}{\alpha}\right)^{n} \frac{\gamma}{2 \alpha|\beta|} \operatorname{det}\left(a_{i \bar{j}}\right)$.

Proof. To prove the claims we apply the above Proposition in a recursive algorithm in three steps. We write $g_{i \bar{j}}$ from (2.4) in the form:

$$
g_{i \bar{j}}=\frac{F}{\alpha}\left(a_{i \bar{j}}-\frac{1}{2 \alpha^{2}} l_{i} l_{\bar{j}}+\frac{\alpha}{2|\beta|} b_{i} b_{\bar{j}}+\frac{\alpha}{2 L F} \eta_{i} \eta_{\bar{j}}\right) .
$$

1) In the first step, we set $Q_{i \bar{j}}:=a_{i \bar{j}}$ and $C_{i}:=\frac{1}{\alpha \sqrt{2}} l_{i}$. By applying the Proposition 2.2 we obtain $Q^{\bar{j} i}=a^{\bar{j} i}, C^{2}=\frac{1}{2}, 1-C^{2}=\frac{1}{2}$ and $C^{i}=\frac{1}{\alpha \sqrt{2}} \eta^{i}$. So, the matrix $H_{i \bar{j}}=a_{i \bar{j}}-\frac{1}{2 \alpha^{2}} l_{i} l_{\bar{j}}$ is invertible with $H^{\bar{j} i}=a^{\bar{j} i}+\frac{1}{\alpha^{2}} \eta^{i} \eta^{\bar{j}}$ and $\operatorname{det}\left(a_{i \bar{j}}-\frac{1}{2 \alpha^{2}} l_{i} l_{\bar{j}}\right)=\frac{1}{2} \operatorname{det}\left(a_{i \bar{j}}\right)$.

2) Now, we consider $Q_{i \bar{j}}:=a_{i \bar{j}}-\frac{1}{2 \alpha^{2}} l_{i} l_{\bar{j}}$ and $C_{i}:=\sqrt{\frac{\alpha}{2|\beta|}} b_{i}$. By applying the Proposition 2.2 we obtain this time:

$$
Q^{\bar{j} i}=a^{\bar{j} i}+\frac{1}{\alpha^{2}} \eta^{i} \bar{\eta}^{j}
$$


and

$$
\begin{gathered}
C^{2}=\frac{\alpha}{2|\beta|}\left(a^{\bar{j} i}+\frac{1}{\alpha^{2}} \eta^{i} \bar{\eta}^{j}\right) b_{i} b_{j}=\frac{\alpha^{2}|| b||^{2}+|\beta|^{2}}{2 \alpha|\beta|} \\
1+C^{2}=\frac{\gamma}{2 \alpha|\beta|} \neq 0
\end{gathered}
$$

$$
C^{i}=\sqrt{\frac{\alpha}{2|\beta|}}\left(a^{\bar{j} i}+\frac{1}{\alpha^{2}} \eta^{i} \bar{\eta}^{j}\right) b_{\bar{j}}=\sqrt{\frac{\alpha}{2|\beta|}}\left(b^{i}+\frac{\bar{\beta}}{\alpha^{2}} \eta^{i}\right) .
$$

It results that the inverse of $H_{i \bar{j}}=a_{i \bar{j}}-\frac{1}{2 \alpha^{2}} l_{i} l_{\bar{j}}+\frac{\alpha}{2|\beta|} b_{i} b_{\bar{j}}$ exists and it is $H^{\bar{j} i}=a^{\bar{j} i}+\frac{1}{\alpha^{2}} \eta^{i} \bar{\eta}^{j}-\frac{\alpha^{2}}{\gamma}\left(b^{i}+\frac{\bar{\beta}}{\alpha^{2}} \eta^{i}\right)\left(\bar{b}^{j}+\frac{\beta}{\alpha^{2}} \bar{\eta}^{j}\right)$, and $\operatorname{det}\left(a_{i \bar{j}}-\frac{1}{2 \alpha^{2}} l_{i} l_{\bar{j}}+\frac{\alpha}{2|\beta|} b_{i} b_{\bar{j}}\right)=\frac{\gamma}{2 \alpha|\beta|} \operatorname{det}\left(a_{i \bar{j}}-\frac{1}{2 \alpha^{2}} l_{i} l_{\bar{j}}\right)=\frac{\gamma}{4 \alpha|\beta|} \operatorname{det}\left(a_{i \bar{j}}\right)$.

3) Finally we put $Q_{i \bar{j}}:=a_{i \bar{j}}-\frac{1}{2 \alpha^{2}} l_{i} l_{\bar{j}}+\frac{\alpha}{2|\beta|} b_{i} b_{\bar{j}}$ and $C_{i}:=\sqrt{\frac{\alpha}{2 L F}} \eta_{i}$. From here, we obtain

$$
\begin{gathered}
Q^{\bar{j} i}=a^{\bar{j} i}+\frac{1}{\alpha^{2}} \eta^{i} \bar{\eta}^{j}-\frac{\alpha^{2}}{\gamma}\left(b^{i}+\frac{\bar{\beta}}{\alpha^{2}} \eta^{i}\right)\left(\bar{b}^{j}+\frac{\beta}{\alpha^{2}} \bar{\eta}^{j}\right), \\
C^{2}=\frac{\alpha}{2 L F}\left[a^{\bar{j} i}+\frac{1}{\alpha^{2}} \eta^{i} \bar{\eta}^{j}-\frac{\alpha^{2}}{\gamma}\left(b^{i}+\frac{\bar{\beta}}{\alpha^{2}} \eta^{i}\right)\left(\bar{b}^{j}+\frac{\beta}{\alpha^{2}} \bar{\eta}^{j}\right)\right] \eta_{i} \eta_{\bar{j}}=1, \\
1+C^{2}=2,
\end{gathered}
$$

and

$C^{i}=\sqrt{\frac{\alpha}{2 L F}}\left[a^{\bar{j} i}+\frac{1}{\alpha^{2}} \eta^{i} \bar{\eta}^{j}-\frac{\alpha^{2}}{\gamma}\left(b^{i}+\frac{\bar{\beta}}{\alpha^{2}} \eta^{i}\right)\left(\bar{b}^{j}+\frac{\beta}{\alpha^{2}} \bar{\eta}^{j}\right)\right] \eta_{\bar{j}}=\frac{2 F}{\alpha} \sqrt{\frac{\alpha}{2 L F}} \eta^{i}$.

By Proposition 2.2 it results that the inverse of

$$
H_{i \bar{j}}=a_{i \bar{j}}-\frac{1}{2 \alpha^{2}} l_{i} l_{\bar{j}}+\frac{\alpha}{2|\beta|} b_{i} b_{\bar{j}}+\frac{\alpha}{2 L F} \eta_{i} \eta_{\bar{j}}
$$

is

$$
H^{\bar{j} i}=a^{\bar{j} i}+\frac{1}{\alpha^{2}} \eta^{i} \bar{\eta}^{j}-\frac{\alpha^{2}}{\gamma}\left(b^{i}+\frac{\bar{\beta}}{\alpha^{2}} \eta^{i}\right)\left(\bar{b}^{j}+\frac{\beta}{\alpha^{2}} \bar{\eta}^{j}\right)-\frac{1}{\alpha F} \eta^{i} \bar{\eta}^{j}
$$

and

$$
\begin{aligned}
\operatorname{det}\left(a_{i \bar{j}}-\frac{1}{2 \alpha^{2}} l_{i} l_{\bar{j}}+\frac{\alpha}{2|\beta|} b_{i} b_{\bar{j}}+\frac{\alpha}{2 L F} \eta_{i} \eta_{\bar{j}}\right) & =2 \operatorname{det}\left(a_{i \bar{j}}-\frac{1}{2 \alpha^{2}} l_{i} l_{\bar{j}}+\frac{\alpha}{2|\beta|} b_{i} b_{\bar{j}}\right) \\
& =\frac{\gamma}{2 \alpha|\beta|} \operatorname{det}\left(a_{i \bar{j}}\right) .
\end{aligned}
$$

But, $g_{i \bar{j}}=\frac{F}{\alpha} H_{i \bar{j}}$, with $H_{i \bar{j}}$ from 3). Thus, $g^{\bar{j} i}=\frac{\alpha}{F} H^{\bar{j} i}$ and $\operatorname{det}\left(g_{i \bar{j}}\right)=$ $\left(\frac{F}{\alpha}\right)^{n} \operatorname{det}\left(H_{i \bar{j}}\right)$. From here, immediately results i) and ii).

Having the formula for $\operatorname{det}\left(g_{i \bar{j}}\right)$, we can say that $g_{i \bar{j}}(z, \eta)$ is positive definite if and only if $\gamma>0$ at each nonzero $\eta$ in $T_{z}^{\prime} M$. So we have proved:

Theorem 2.1. A complex Randers metric with $\gamma>0$ is a complex Finsler metric. 
If the quadratic form $h(z, \eta):=\left(a_{i \bar{j}}-b_{i} b_{\bar{j}}\right) \eta^{i} \bar{\eta}^{j}$ is positive definite, then substituting $\eta^{i} \bar{\eta}^{j}$ with $b^{i} \bar{b}^{j}$ it follows that $\|b\|^{2}\left(1-\|b\|^{2}\right)>0$, which says that $\|\left. b\right|^{2} \in(0,1)$ and then $\gamma>0$, since $\gamma=2 \alpha|\beta|+|\beta|^{2}+\left.\alpha^{2}|| b\right|^{2}$. Equivalently the positive definite of the quadratic form means that $\alpha^{2}>|\beta|^{2}$, or in other words $\sup \frac{|\beta|}{\alpha}<1$ for all $(z, \eta) \in T^{\prime} M \backslash\{0\}$. The last assumption is required in [20] and no other restrictive conditions are needful for a complex Randers metric to be a complex Finsler metric.

Example 2.1. If $\alpha(z, \eta)=m c \sqrt{\gamma_{i \bar{j}}(z) \eta^{i} \bar{\eta}^{j}}$ and $\beta(z, \eta)=\frac{e}{m} A_{i}(z) \eta^{i}$, where $m$, $c, e$ are real scalars, a model for complex electrodynamics is obtain.

Example 2.2. We consider $\alpha$ given by

$$
\alpha^{2}(z, \eta):=\frac{|\eta|^{2}+\varepsilon\left(|z|^{2}|\eta|^{2}-|\langle z, \eta\rangle|^{2}\right)}{\left(1+\varepsilon|z|^{2}\right)^{2}},
$$

where $|z|^{2}:=\sum_{k=1}^{n} z^{k} \bar{z}^{k},\langle z, \eta\rangle:=\sum_{k=1}^{n} z^{k} \bar{\eta}^{k},|\langle z, \eta\rangle|^{2}=\langle z, \eta\rangle \overline{\langle z, \eta\rangle}$, defined over the disk $\Delta_{r}^{n}=\left\{z \in \mathbb{C}^{n},|z|<r, \quad r:=\sqrt{\frac{1}{|\varepsilon|}}\right\}$ if $\varepsilon<0$, on $\mathbb{C}^{n}$ if $\varepsilon=0$ and on the complex projective space $P^{n}(\mathbb{C})$ if $\varepsilon>0$. Note that $\alpha^{2}(z, \eta)=a_{i \bar{j}}(z) \eta^{i} \bar{\eta}^{j}$ and thus determines purely Hermitian metrics which have special properties. They are Kähler with constant holomorphic curvature $\mathcal{K}_{\alpha}=4 \varepsilon$. Particularly, for $\varepsilon=-1$ we obtain the Bergman metric on the unit disk $\Delta^{n}:=\Delta_{1}^{n}$; for $\varepsilon=0$ the Euclidean metric on $\mathbb{C}^{n}$, and for $\varepsilon=1$ the Fubini-Study metric on $P^{n}(\mathbb{C})$.

By deformation of (2.5) metrics, taking $|\beta(z, \eta)|=\frac{|\langle z, \eta\rangle|}{1+\varepsilon|z|^{2}}$ we obtain some examples of complex Randers metrics:

$$
F_{\varepsilon}:=\frac{\sqrt{|\eta|^{2}+\varepsilon\left(|z|^{2}|\eta|^{2}-|\langle z, \eta\rangle|^{2}\right)}}{1+\varepsilon|z|^{2}}+\frac{|\langle z, \eta\rangle|}{1+\varepsilon|z|^{2}} .
$$

For example, $F_{-1}$ is of negative holomorphic curvature $\mathcal{K}_{F_{-1}}=\frac{-2 \alpha F_{-1}}{\gamma}$, $\gamma:=L_{-1}-\alpha^{2}\left(1-|z|^{2}\right)$.

Further we show that the complex Randers metric $F:=\alpha+|\beta|$ offers a significant $d$-tensor with fairly many properties. Let us consider

$$
k_{i \bar{j}}:=\frac{\partial^{2} F}{\partial \eta^{i} \partial \bar{\eta}^{j}}=\frac{1}{2 F}\left(g_{i \bar{j}}-\frac{\partial F}{\partial \eta^{i}} \frac{\partial F}{\partial \bar{\eta}^{j}}\right)=\frac{1}{2 F}\left(g_{i \bar{j}}-\frac{1}{2 L} \eta_{i} \eta_{\bar{j}}\right) .
$$

We call (2.7) the complex angular metric tensor of the space. A direct computation yields $k_{i \bar{j}} \eta^{i}=\frac{1}{4 F} \eta_{\bar{j}}, k_{i \bar{j}} \eta^{i} \bar{\eta}^{j}=\frac{F}{4}, \frac{\partial k_{i \bar{j}}}{\partial \eta^{m}} \eta^{m}=-\frac{1}{2} k_{i \bar{j}}$. Moreover we have:

Proposition 2.4. i) $k_{i \bar{j}}=\frac{1}{2 \alpha} h_{i \bar{j}}+\frac{1}{4|\beta|} b_{i} b_{\bar{j}}$;

ii) $\left(k_{i \bar{j}}\right)$ is invertible and its inverse is

$$
k^{\bar{j} i}=2 \alpha a^{\bar{j} i}+\frac{2\left(\alpha \|\left. b\right|^{2}+2|\beta|\right)}{\gamma} \eta^{i} \bar{\eta}^{j}-\frac{2 \alpha^{3}}{\gamma} b^{i} \bar{b}^{j}-\frac{2 \alpha}{\gamma}\left(\bar{\beta} \eta^{i} \bar{b}^{j}+\beta b^{i} \bar{\eta}^{j}\right) ;
$$


iii) $\operatorname{det}\left(k_{i \bar{j}}\right)=\frac{\gamma}{2(2 \alpha)^{n+1}|\beta|} \operatorname{det}\left(a_{i \bar{j}}\right)$.

The proof is straightforward, applying Proposition 2.2.

Once obtained the metric tensor of a complex Randers space, it is a technical computation to get the expression of (1.1) Chern-Finsler connection. Certainly, it involves a lot of trivial calculus and for this reason we will not dwell too much on it. Computational details can be found in [5].

The first computation refers to the coefficients of the Chern-Finsler (c.n.c.). A simplified writing for them is

$$
\stackrel{C F}{N_{j}^{i}}=\stackrel{a}{=} N_{j}^{i}+\frac{1}{\gamma}\left(l_{\bar{r}} \frac{\partial b^{\bar{r}}}{\partial z^{j}}-\frac{\beta^{2}}{|\beta|^{2}} \frac{\partial b_{\bar{r}}}{\partial z^{j}} \bar{\eta}^{r}\right) \xi^{i}+\frac{\beta}{2|\beta|} k^{\bar{r} i} \frac{\partial b_{\bar{r}}}{\partial z^{j}},
$$

where $\xi^{i}:=\bar{\beta} \eta^{i}+\alpha^{2} b^{i}$ and $\stackrel{a}{N_{j}^{i}}:=a^{\bar{m} i} \frac{\partial a_{l \bar{m}}}{\partial z^{j}} \eta^{l}$.

Next, let us introduce the following complex Cartan tensors $([3])$ :

$$
C_{j \bar{h} k}:=\frac{\partial g_{j \bar{h}}}{\partial \eta^{k}}=\frac{\alpha|\beta|}{4 \delta}\left(\frac{1}{\alpha^{4}} l_{j} l_{k}-\frac{\bar{\beta}^{2}}{|\beta|^{4}} b_{j} b_{k}\right) C_{\bar{h}}-\frac{|\beta|}{2 \delta \alpha}\left(r_{j \bar{h}} C_{k}+r_{k \bar{h}} C_{j}\right)
$$

where $C_{j}:=\delta\left(\frac{1}{\alpha^{2}} l_{j}-\frac{\bar{\beta}}{|\beta|^{2}} b_{j}\right)=C_{j \bar{h} k} g^{\bar{h} k} ; \delta:=\frac{\alpha^{2}|| b||^{2}-|\beta|^{2}}{2 \gamma}-\frac{n|\beta|}{2 F}$ and $r_{j \bar{h}}:=$ $h_{j \bar{h}}-\frac{\alpha^{2}}{2|\beta|^{2}} b_{j} b_{\bar{h}}$, with $h_{j \bar{h}}$ from (2.4).

Then the vertical coefficients of Chern-Finsler connections are

$$
C_{j k}^{i}:=g^{\bar{m} i} \frac{\partial g_{k \bar{m}}}{\partial \eta^{j}}=g^{\bar{m} i} \frac{\partial g_{j \bar{m}}}{\partial \eta^{k}}=g^{\bar{m} i} C_{j \bar{m} k} .
$$

An expanded writing for these coefficients is

$$
\begin{aligned}
(2.10) C_{j k}^{i}= & \frac{\alpha|\beta|}{2 \gamma}\left(\frac{1}{\alpha^{4}} l_{j} l_{k}-\frac{\bar{\beta}^{2}}{|\beta|^{4}} b_{j} b_{k}\right) \varepsilon^{i} \\
& -\frac{|\beta|}{2 \delta F}\left(\delta_{j}^{i} C_{k}+\delta_{k}^{i} C_{j}\right)+\frac{1}{2 \delta \gamma}\left(b_{j} C_{k}+b_{k} C_{j}\right) \xi^{i}-\frac{1}{L} C_{j k} \eta^{i},
\end{aligned}
$$

where $\varepsilon^{i}:=\frac{\alpha|| b||^{2}+|\beta|}{F} \eta^{i}-\frac{\alpha \beta}{|\beta|} b^{i}$ and $C_{j k}:=C_{j \bar{h} k} \bar{\eta}^{h}=-\frac{|\beta| \alpha}{2 \delta^{2}} C_{j} C_{k}$.

We remark that a complex Finsler metric is purely Hermitian if and only if $C_{k}=0$. For a complex Randers metric, $C_{k}=0$ leads to $F=\alpha(1+\|b\|)$ and $a_{i \bar{j}}\|b\|^{2}=b_{i} b_{\bar{j}}$. Thus, we have proved:

Colorallary 2.1. A complex Randers metric is purely Hermitian if and only if $a_{i \bar{j}}\|b\|^{2}=b_{i} b_{\bar{j}}$.

For the horizontal coefficients $L_{j k}^{i}=\frac{\partial N_{k}^{i}}{\partial \eta^{j}}$ of the Chern-Finsler connection, and for the (1.2) holomorphic curvature of the complex Randers spaces $(M, \alpha+$ $|\beta|)$ in direction $\eta$, the calculus are a bit intricate and can be found in [5], and therefore here we pass over these. 
Up to this point the above discussions are in some analogies with the real Randers case. Indeed the study of some geometrical aspects on complex Randers metrics is of interest.

\section{Kähler-Randers metrics}

When trying to show more geometrical properties of complex Randers metrics, we face the fact that there are so many computations. Certainly, one should not infer that this class of complex Finsler metrics is less significant. On the contrary, beyond the computations in the sequel we show that there are interesting results.

Proposition 3.1. Let $(M, \alpha+|\beta|)$ be a complex Randers space with property $\frac{\partial|\beta|^{2}}{\partial z^{i}}=\varepsilon \frac{\partial \alpha^{2}}{\partial z^{i}}$, where $\varepsilon=\varepsilon(z)$ is a real valued function.

i) If $a_{i \bar{j}}$ is the Euclidian metric, then $F=\alpha+|\beta|$ is locally Minkowski.

ii) If $\varepsilon=0$ for any $z, C_{k} \neq 0$ for any $k$, and $a_{i \bar{j}}$ is Kähler, then $F=\alpha+|\beta|$ is weakly Kähler if and only if $b_{i} \stackrel{a}{G^{i}}=0$, where $\stackrel{a}{G^{i}}:=\stackrel{a}{N_{j}^{i}} \eta^{j}$.

iii) If $\varepsilon=\|b\|^{2}$, then $\|b\|^{2}$ is a constant.

Proof. Indeed, if $a_{i \bar{j}}$ is the Euclidian metric, then $\frac{\partial|\beta|^{2}}{\partial z^{i}}=\frac{\partial \alpha^{2}}{\partial z^{i}}=0$ and so $\frac{\partial F}{\partial z^{i}}=0$. This means that there exist charts in any $(z, \eta)$ such that the complex Randers metric $F=\alpha+|\beta|$ depends only on the $\eta$ variable, i.e., it is locally Minkowski and i) is true.

The assumption $\frac{\partial|\beta|^{2}}{\partial z^{i}}=\varepsilon \frac{\partial \alpha^{2}}{\partial z^{i}}$ can be written as

$$
\bar{\beta} \frac{\partial b_{r}}{\partial z^{i}} \eta^{r}+\beta \frac{\partial b_{\bar{m}}}{\partial z^{i}} \bar{\eta}^{m}=\varepsilon \frac{\partial a_{r \bar{m}}}{\partial z^{i}} \eta^{r} \bar{\eta}^{m} .
$$

Deriving the relation (3.1) with respect to $\eta$ and $\bar{\eta}$, we obtain

$$
b_{\bar{m}} \frac{\partial b_{r}}{\partial z^{i}}+b_{r} \frac{\partial b_{\bar{m}}}{\partial z^{i}}=\varepsilon \frac{\partial a_{r \bar{m}}}{\partial z^{i}} .
$$

Now, contracting in (3.2) by $b^{r}$ and $b^{\bar{m}}$, we get

$$
b^{r} \frac{\partial b_{r}}{\partial z^{i}}+b^{\bar{m}} \frac{\partial b_{\bar{m}}}{\partial z^{i}}=\frac{\varepsilon}{\|b\|^{2}} b^{\bar{m}} b^{r} \frac{\partial a_{r \bar{m}}}{\partial z^{i}} .
$$

In [5], after the calculus of the $L_{j k}^{i}$ coefficients we obtain the weakly Kähler condition for a complex Randers space. By this, a straightforward computation using (3.3) with $\varepsilon=0$ and $\Gamma_{\bar{j} k}^{\bar{r}}=\frac{1}{2} a^{\bar{r} k}\left\{\frac{\partial a_{k \bar{j}}}{\partial z^{i}}-\frac{\partial a_{\bar{j}}}{\partial z^{k}}\right\}=0$ since $a_{i \bar{j}}$ is Kähler, show immediate that the assertion ii) is true.

Now, by (3.3) we obtain $\frac{\partial\|b\|^{2}}{\partial z^{i}}=-\left(1-\frac{\varepsilon}{\|b\|^{2}}\right) b^{\bar{m}} b^{r} \frac{\partial a_{r \bar{m}}}{\partial z^{i}}$. Putting $\varepsilon=\|b\|^{2}$ in last relation we have $\frac{\partial\|b\|^{2}}{\partial z^{i}}=0$, and so $\|b\|^{2}$ is a constant, i.e., iii).

Lemma 3.1. Let $(M, \alpha+|\beta|)$ be a complex Randers space with property $\frac{\partial|\beta|^{2}}{\partial z^{i}}=$ $\|b\|^{2} \frac{\partial \alpha^{2}}{\partial z^{i}}$. The following statements are equivalent: 
i) $\|b\|^{2} b^{r} \frac{\partial a_{r \bar{m}}}{\partial z^{l}} \bar{\eta}^{m}=\bar{\beta} b^{\bar{m}} b^{r} \frac{\partial a_{r \bar{m}}}{\partial z^{l}}$;

ii) $\|b\|^{2} \frac{\partial b_{\bar{m}}}{\partial z^{i}} \bar{\eta}^{m}=\bar{\beta} \frac{\partial b_{\bar{m}}}{\partial z^{i}} b^{\bar{m}}$;

iii) $b_{\bar{s}} \frac{\partial b_{\bar{m}}}{\partial z^{i}} \bar{\eta}^{m}=\bar{\beta} \frac{\partial b_{\bar{s}}}{\partial z^{i}}$;

iv) $\bar{\beta}\left(\frac{\partial b_{i}}{\partial z^{l}} \eta^{i} \eta^{l}-b_{l} G^{l}\right)+\beta \frac{\partial b_{\bar{m}}}{\partial z^{l}} \bar{\eta}^{m} \eta^{l}=0$.

Proof. i) $\Longleftrightarrow$ ii). A straightforward computation, using Proposition 3.1 iii), lead us to

$$
\|b\|^{2} \frac{\partial b_{\bar{m}}}{\partial z^{i}} \bar{\eta}^{m}-\bar{\beta} \frac{\partial b_{\bar{m}}}{\partial z^{i}} b^{\bar{m}}=\|b\|^{2} b^{r} \frac{\partial a_{r \bar{m}}}{\partial z^{l}} \bar{\eta}^{m}-\bar{\beta} b^{\bar{m}} b^{r} \frac{\partial a_{r \bar{m}}}{\partial z^{l}} .
$$

By (3.4), immediately results the equivalence between i) and ii).

ii) $\Longleftrightarrow$ iii). Given ii), deriving it with respect to $\bar{\eta}$, we obtain $\|b\|^{2} \frac{\partial b_{\bar{s}}}{\partial z^{i}}=$ $b_{\bar{s}} \frac{\partial b_{\bar{m}}}{\partial z^{i}} b^{\bar{m}}$. Using again ii) we obtain iii). Conversely, contracting iii) by $b^{\bar{s}}$ it results ii).

ii) $\Longleftrightarrow$ iv). Now, deriving the relation (3.1) with respect to $\bar{\eta}$ and contracting it by $b^{\bar{m}}$ we deduce

$$
\|b\|^{2} \bar{\beta}\left(\frac{\partial b_{i}}{\partial z^{l}} \eta^{i} \eta^{l}-b_{l} \stackrel{a}{G}^{l}\right)+|\beta|^{2} \frac{\partial b_{\bar{m}}}{\partial z^{i}} b^{\bar{m}} \eta^{l}=0,
$$

and the equivalence between assumptions ii) and iv) is trivial. So, the proof is complete.

Proposition 3.2. Let $(M, \alpha+|\beta|)$ be a complex Randers space with $\frac{\partial|\beta|^{2}}{\partial z^{i}}=$ $\|b\|^{2} \frac{\partial \alpha^{2}}{\partial z^{i}}$. If one of equivalent conditions from Lemma 3.1 holds, then $N_{j}^{i}=N_{j}^{i}$. Proof. An elementary computation, taking into account Lemma 3.1 in the formula (2.8) which give the coefficients of Chern-Finsler (c.n.c), prove that all terms are vanishes except the first term. Indeed we obtain $N_{j}^{i}=\stackrel{a}{i} N_{j}^{i}$.

Proposition 3.3. Let $(M, \alpha+|\beta|)$ be a complex Randers space with $\frac{\partial|\beta|^{2}}{\partial z^{i}}=$ $\|b\|^{2} \frac{\partial \alpha^{2}}{\partial z^{i}}$ and $a_{i \bar{j}}$ be Kähler. If one of equivalent conditions from Lemma 3.1 holds, then $F=\alpha+|\beta|$ is strongly Kähler.

Proof. By Proposition 3.2, we have $\stackrel{C F}{N_{j}^{i}}=\stackrel{a}{N_{j}^{i}}$ and $L_{j k}^{i}=\frac{\stackrel{C F}{i}}{\partial N_{k}^{i}}=\frac{\partial N_{k}^{i}}{\partial \eta^{j}}$. Because $a_{i \bar{j}}$ is Kähler, it results $\frac{\partial N_{k}^{i}}{\partial \eta^{j}}=\frac{\partial N_{j}^{i}}{\partial \eta^{k}}$. Therefore, $L_{j k}^{i}=L_{k j}^{i}$, i.e., $F=\alpha+|\beta|$ is strongly Kähler.

Finally, we depict some examples which illustrate our theory.

Example 3.1. We consider $a_{i \bar{j}}=\frac{1}{1-|z|^{2}}\left(\delta_{i \bar{j}}+\frac{\bar{z}^{i} z^{j}}{1-|z|^{2}}\right)$ the Bergman metric on the unit disk $\Delta^{n}:=\left\{z \in \mathbb{C}^{n},|z|<1\right\}$ which is a Kähler-purely Hermitian 
metric, of holomorphic curvature -4 . In order to obtain an example of strongly Kähler-Randers metric, we must to solve the system of differential equations

$$
\|b\|^{2} b^{r} \frac{\partial a_{r \bar{m}}}{\partial z^{l}} \bar{\eta}^{m}=\bar{\beta} b^{\bar{m}} b^{r} \frac{\partial a_{r \bar{m}}}{\partial z^{l}},
$$

that is the assumptions i) from Lemma 3.1.

A straightforward computation, using $\|b\|^{2}=\left(1-|z|^{2}\right)\left(b_{i} b_{\bar{\imath}}-\bar{z}^{i} b_{\bar{\imath}} z^{k} b_{k}\right)$ gives:

$$
\bar{z}^{i} b_{\bar{\imath}}\left(a_{l \bar{j}}-\frac{1}{\|b\|^{2}} b_{l} b_{\bar{j}}\right)=0 .
$$

So, we obtain two cases:

a) $a_{l \bar{j}}=\frac{1}{\|b\|^{2}} b_{l} b_{\bar{j}}$. This means that the Randers metric is $F=\alpha+|\beta|=$ $\alpha(1+\|b\|)$, and hence the space is with purely Hermitian metric, or

b) $b_{i} z^{i}=0$ which leads to a strongly Kähler-Randers metric $F=\alpha+|\beta|$ on $\Delta^{n}$ with properties

$$
\begin{array}{rl}
b_{i} b_{\bar{\imath}} & =\frac{\|b\|^{2}}{1-|z|^{2}} ; \quad b_{i}=-\frac{\partial b_{l}}{\partial z^{i}} z^{l} ; \frac{\partial b_{\bar{l}}}{\partial z^{i}} \bar{z}^{l}=0 ; \\
C F & a \\
N_{j}^{i} & =N_{j}^{i}=\frac{1}{1-|z|^{2}}\left(\bar{z}^{j} \eta^{i}+\bar{z}^{k} \eta^{k} \delta_{j}^{i}\right) ; \mathcal{K}_{F}=-\frac{4 \alpha^{2}}{L}<0 .
\end{array}
$$

Example 3.2. Now, we give an example of complex Randers space of complex dimension two. In order to reduce clutter, let us relabel the local coordinates $z^{1}, z^{2}, \eta^{1}, \eta^{2}$ as $z, w, \eta, \theta$, respectively.

Let

$$
D=\left\{(z, w) \in \mathbf{C}^{2},|w|<|z|<1\right\}
$$

be the Hartogs triangle with the Kähler-purely Hermitian metric

$$
a_{i \bar{j}}=\frac{\partial^{2}}{\partial z^{i} \partial \bar{z}^{j}}\left(\log \frac{1}{\left(1-|z|^{2}\right)\left(|z|^{2}-|w|^{2}\right)}\right) ; \alpha^{2}(z, w ; \eta, \theta)=a_{i \bar{j}} \eta^{i} \bar{\eta}^{j}
$$

where $\left|z^{i}\right|^{2}:=z^{i} \bar{z}^{i}, z^{i} \in\{z, w\}, \eta^{i} \in\{\eta, \theta\}$. We choose

$$
b_{z}=\frac{w}{|z|^{2}-|w|^{2}} ; b_{w}=-\frac{z}{|z|^{2}-|w|^{2}} .
$$

By a direct computation, we deduce

$$
\begin{aligned}
& a_{z \bar{z}}=\frac{1}{\left(1-|z|^{2}\right)^{2}}+b_{z} b_{\bar{z}} ; a_{z \bar{w}}=b_{z} b_{\bar{w}} ; a_{w \bar{w}}=b_{w} b_{\bar{w}} \\
& a^{\bar{z} z}=\left(1-|z|^{2}\right)^{2} ; a^{\bar{w} z}=\frac{\bar{w} z\left(1-|z|^{2}\right)^{2}}{|z|^{2}}
\end{aligned}
$$




$$
\begin{aligned}
a^{\bar{w} w} & =\frac{\left(|z|^{2}-|w|^{2}\right)^{2}}{|z|^{2}}+\frac{|w|^{2}\left(1-|z|^{2}\right)^{2}}{|z|^{2}} ; \\
b^{z} & =0 ; b^{w}=-\frac{|z|^{2}-|w|^{2}}{z} ; \\
\|b\|^{2} & =1 ; \alpha^{2}-|\beta|^{2}=\frac{|\eta|^{2}}{\left(1-|z|^{2}\right)^{2}}
\end{aligned}
$$

and the coefficients of the Chern-Finsler (c.n.c.) are

$$
\begin{aligned}
& \stackrel{C F}{N_{z}^{z}}=\stackrel{a}{N_{z}^{z}}=\frac{2 \bar{z} \eta}{1-|z|^{2}} ; \stackrel{C F}{N_{w}^{z}}=\stackrel{a}{N_{w}^{z}}=0 \\
& { }_{\text {CF }}^{\text {N }}=\stackrel{a}{N_{z}^{w}}=\frac{2 \bar{z} w}{z}\left(\frac{1}{1-|z|^{2}}+\frac{1}{|z|^{2}-|w|^{2}}\right) \eta-\frac{|z|^{2}+|w|^{2}}{z\left(|z|^{2}-|w|^{2}\right)} \theta \\
& \stackrel{C}{N_{w}^{w}}=\stackrel{a}{N_{w}^{w}}=-\frac{|z|^{2}+|w|^{2}}{z\left(|z|^{2}-|w|^{2}\right)} \eta+\frac{2 \bar{w} \theta}{|z|^{2}-|w|^{2}}
\end{aligned}
$$

Thus we have another example of strongly Kähler-Randers metric. It is a complex Finsler metric on Hartogs triangle and its holomorphic curvature is negative, $\mathcal{K}_{F}=-\frac{4}{L \alpha}\left[F(\alpha-|\beta|)^{2}+|\beta|^{3}\right]<0$.

\section{The variational problem}

The variational problem for a complex Lagrangian $L=F^{2}$, with its fundamental metric tensor $g_{i \bar{j}}=\frac{\partial^{2} L}{\partial \eta^{i} \partial \bar{\eta}^{j}}$, lead in [15, p. 94], to a complex geodesic curve, which is an horizontal curve in weakly Kähler circumstances. On the other hand this complex geodesic determines two complex nonlinear connections depending only on the complex Lagrangian $L$, one is the Car$\tan ($ c.n.c.) and the other is the Chern-Lagrange (c.n.c.) (in particular ChernFinsler (c.n.c.)), both related to the same complex spray. The Chern-Finsler (c.n.c.) is precisely determined when we try to lift a Hermitian vertical connection to the whole complexified tangent bundle of $T^{\prime} M$ manifold (good vertical connection in [1]). Certainly, the Chern-Finsler (c.n.c.) has its geometrical meaning but from a physical point of view, and note that complex Lagrange geometry is a model for many quantum physics theories ([15]), we should not loose interest in other (c.n.c.) intrinsically related to the variational problem for a complex Randers Lagrangian.

Following some ideas from the real case ([14]), we shall study the variational problem for the Lagrangian $L=F^{2}=(\alpha+|\beta|)^{2}$ in the canonical parametrization of a curve on complex manifold $M$ with respect to the purely Hermitian structure $\alpha$. The outcome is a (c.n.c.) of Lorentz type in the canonical parametrization.

Let us consider $c(t), t \in \mathbb{R}$, a $C^{\infty}$ curve on complex manifold $M$ and $\left(z^{k}(t), \eta^{k}(t)=\frac{d z^{k}}{d t}\right)$ its extension on $T^{\prime} M$. The Euler-Lagrange equations with 
respect to a complex Lagrangian $L$ are $([2,15,17])$

$$
E_{i}(L):=\frac{\partial L}{\partial z^{i}}-\frac{d}{d t}\left(\frac{\partial L}{\partial \eta^{i}}\right)=0
$$

where $L$ is considered along the curve $c$ on $T^{\prime} M$. Generally, the solutions of the Euler-Lagrange equations are extremal curves which respect to arc length.

Proposition 4.1. For any complex Finsler space $(M, F)$ we have $\frac{d L}{d t}=\frac{d F}{d t}=$ 0 , along an extremal curve $c$ on $T^{\prime} M$.

Proof. First, taking into account that $L=(\alpha+|\beta|)^{2}$ depends on the parameter $t \in \mathbb{R}$ by means of $z^{k}(t), \eta^{k}(t)$ and theirs conjugates, we have

$$
\frac{d L}{d t}=\frac{\partial L}{\partial z^{i}} \eta^{i}+\frac{\partial L}{\partial \eta^{i}} \frac{d \eta^{i}}{d t}+\frac{\partial L}{\partial \bar{z}^{i}} \bar{\eta}^{i}+\frac{\partial L}{\partial \bar{\eta}^{i}} \frac{d \bar{\eta}^{i}}{d t} .
$$

Then, from the homogeneity of Finsler function we have $\frac{\partial L}{\partial \eta^{i}} \eta^{i}=L$ and hence $\frac{d L}{d t}=\frac{d}{d t}\left(\frac{\partial L}{\partial \eta^{i}}\right) \eta^{i}+\frac{\partial L}{\partial \eta^{i}} \frac{d \eta^{i}}{d t}$. But $E_{i}(L)=0$ says $\frac{d}{d t}\left(\frac{\partial L}{\partial \eta^{i}}\right)=\frac{\partial L}{\partial z^{i}}$ along the extremal curve $c$ on $T^{\prime} M$. It follows then $\frac{d L}{d t}=\frac{\partial L}{\partial z^{i}} \eta^{i}+\frac{\partial L}{\partial \eta^{i}} \frac{d \eta^{i}}{d t}$ and, since $t$ and $L$ are real valued, by conjugation we obtain $\frac{d L}{d t}=\frac{\partial L}{\partial \bar{z}^{i}} \bar{\eta}^{i}+\frac{\partial L}{\partial \bar{\eta}^{i}} \frac{d \bar{\eta}^{i}}{d t}$. Summing up the last two relations, and looking at (1) formula, we get $\frac{d L}{d t}=0$. Moreover, $\frac{d L}{d t}=\frac{d F^{2}}{d t}=2 F \frac{d F}{d t}=0$ implies $\frac{d F}{d t}=0$.

Let us develop the calculus in (4.1) for $L=(\alpha+|\beta|)^{2}$ :

$$
\begin{aligned}
\frac{\partial L}{\partial z^{i}} & =L_{\alpha} \frac{\partial \alpha}{\partial z^{i}}+L_{|\beta|} \frac{\partial|\beta|}{\partial z^{i}}=2 F\left(\frac{\partial \alpha}{\partial z^{i}}+\frac{\partial|\beta|}{\partial z^{i}}\right) \\
\frac{\partial L}{\partial \eta^{i}} & =L_{\alpha} \frac{\partial \alpha}{\partial \eta^{i}}+L_{|\beta|} \frac{\partial|\beta|}{\partial \eta^{i}}=2 F\left(\frac{\partial \alpha}{\partial \eta^{i}}+\frac{\partial|\beta|}{\partial \eta^{i}}\right) ; \\
\frac{d}{d t}\left(\frac{\partial L}{\partial \eta^{i}}\right) & =\frac{d L_{\alpha}}{d t} \frac{\partial \alpha}{\partial \eta^{i}}+L_{\alpha} \frac{d}{d t}\left(\frac{\partial \alpha}{\partial \eta^{i}}\right)+\frac{d L_{|\beta|}}{d t} \frac{\partial|\beta|}{\partial \eta^{i}}+L_{|\beta|} \frac{d}{d t}\left(\frac{\partial|\beta|}{\partial \eta^{i}}\right) \\
& =2 \frac{d F}{d t}\left(\frac{\partial \alpha}{\partial \eta^{i}}+\frac{\partial|\beta|}{\partial \eta^{i}}\right)+2 F\left[\frac{d}{d t}\left(\frac{\partial \alpha}{\partial \eta^{i}}\right)+\frac{d}{d t}\left(\frac{\partial|\beta|}{\partial \eta^{i}}\right)\right] \\
& =2 F\left[\frac{d}{d t}\left(\frac{\partial \alpha}{\partial \eta^{i}}\right)+\frac{d}{d t}\left(\frac{\partial|\beta|}{\partial \eta^{i}}\right)\right]
\end{aligned}
$$

along the extremal curve $c$ on $T^{\prime} M$, in view of Proposition 2.1.

Thus, (4.1) yields $E_{i}(L)=2 F\left(E_{i}(\alpha)+E_{i}(|\beta|)\right)=0$. But,

$$
E_{i}\left(\alpha^{2}\right)=\frac{\partial \alpha^{2}}{\partial z^{i}}-\frac{d}{d t}\left(\frac{\partial \alpha^{2}}{\partial \eta^{i}}\right)=2 \alpha \frac{\partial \alpha}{\partial z^{i}}-2 \frac{d}{d t}\left(\alpha \frac{\partial \alpha}{\partial \eta^{i}}\right)=2 \alpha E_{i}(\alpha)-2 \frac{d \alpha}{d t} \frac{\partial \alpha}{\partial \eta^{i}}
$$


and

$$
\begin{aligned}
E_{i}\left(|\beta|^{2}\right) & =\frac{\partial|\beta|^{2}}{\partial z^{i}}-\frac{d}{d t}\left(\frac{\partial|\beta|^{2}}{\partial \eta^{i}}\right)=2|\beta| \frac{\partial|\beta|}{\partial z^{i}}-2 \frac{d}{d t}\left(|\beta| \frac{\partial|\beta|}{\partial \eta^{i}}\right) \\
& =2|\beta| E_{i}(|\beta|)-2 \frac{d|\beta|}{d t} \frac{\partial|\beta|}{\partial \eta^{i}} .
\end{aligned}
$$

So, we have proved:

Proposition 4.2. The Euler-Lagrange equations with respect to $L=(\alpha+|\beta|)^{2}$ are

$$
|\beta| E_{i}\left(\alpha^{2}\right)+\alpha E_{i}\left(|\beta|^{2}\right)+2|\beta| \frac{d \alpha}{d t} \frac{\partial \alpha}{\partial \eta^{i}}+2 \alpha \frac{d|\beta|}{d t} \frac{\partial|\beta|}{\partial \eta^{i}}=0
$$

Now, we choose $s(t)$ the arc length of the curve $c$ on $T^{\prime} M$ with respect to the purely Hermitian structure $\alpha$ as a parametrization of the curve $c$ on $T^{\prime} M$. Since $d s^{2}=\alpha^{2}\left(z, \frac{d z}{d t}\right) d t^{2}$ it yields $\alpha^{2}\left(z, \frac{d z}{d s}\right)=1$ and hence

$$
\frac{d \alpha}{d s}=0 ; \eta^{i}=\frac{d z^{i}}{d t}=\frac{d z^{i}}{d s} \frac{d s}{d t}=\alpha \frac{d z^{i}}{d s} \text { and } \frac{d \eta^{i}}{d s}=\alpha \frac{d^{2} z^{i}}{d s^{2}}
$$

In Proposition 3.1 we assert that $\frac{d F}{d t}=0$ with $F=\alpha+|\beta|$. Since $\frac{d \alpha}{d s}=0$ in the canonical parametrization, it results that $\frac{d|\beta|}{d s}=0$, along the extremal curve $c$ on $T^{\prime} M$. As a consequence of (4.2) we have:

Theorem 4.1. For the complex Randers spaces $(M, \alpha+|\beta|)$, the Euler-Lagrange equations in the canonical parametrization are

$$
|\beta| E_{i}\left(\alpha^{2}\right)+\alpha E_{i}\left(|\beta|^{2}\right)=0 .
$$

By analogy with the real case, [14], we call the equations (4.4) the Lorentz equations of the complex Randers space $(M, \alpha+|\beta|)$.

Next, let us compute $E_{i}\left(\alpha^{2}\right)$ and $E_{i}\left(|\beta|^{2}\right)$ in the canonical parametrization. First of all, the condition $\frac{d|\beta|}{d s}=0$ is equivalent to

$$
\alpha^{2} b_{\bar{l}} \frac{d^{2} \bar{z}^{l}}{d s^{2}}=-q_{\bar{l}} \bar{\eta}^{l}-\frac{\bar{\beta}^{2}}{|\beta|^{2}}\left(q_{l} \eta^{l}+\alpha^{2} b_{l} \frac{d^{2} z^{l}}{d s^{2}}\right),
$$

which multiplied with $b_{i}$ gives

$$
-\alpha^{2} b_{i} b_{\bar{l}} \frac{d^{2} \bar{z}^{l}}{d s^{2}}=b_{i} q_{\bar{l}} \bar{\eta}^{l}+\frac{\bar{\beta}^{2}}{|\beta|^{2}} b_{i} q_{l} \eta^{l}+\frac{\bar{\beta}^{2} \alpha^{2}}{|\beta|^{2}} b_{i} b_{l} \frac{d^{2} z^{l}}{d s^{2}}
$$

where $q_{l}:=\frac{\partial b_{l}}{\partial z^{r}} \eta^{r}+\frac{\partial b_{l}}{\partial \bar{z}^{r}} \bar{\eta}^{r} ; \quad \overline{q_{\bar{l}}}=q_{l}$.

We have

$$
\begin{aligned}
E_{i}\left(\alpha^{2}\right) & =\frac{\partial \alpha^{2}}{\partial z^{i}}-\frac{d}{d t}\left(\frac{\partial \alpha^{2}}{\partial \eta^{i}}\right)=\frac{\partial \alpha^{2}}{\partial z^{i}}-\frac{d}{d s}\left(\frac{\partial \alpha^{2}}{\partial \eta^{i}}\right) \frac{d s}{d t} \\
& =-\alpha^{2} a_{i \bar{l}} \frac{d^{2} \bar{z}^{l}}{d s^{2}}+2 a_{l \bar{r}} \Gamma_{j i}^{\bar{r}} \bar{\eta}^{j} \eta^{l}-a_{i \bar{r}} \Gamma_{\bar{j} \bar{l}}^{\bar{r}} \bar{\eta}^{j} \bar{\eta}^{l} ;
\end{aligned}
$$




$$
\begin{aligned}
& E_{i}\left(|\beta|^{2}\right) \\
= & \frac{\partial|\beta|^{2}}{\partial z^{i}}-\frac{d}{d t}\left(\frac{\partial|\beta|^{2}}{\partial \eta^{i}}\right)=\frac{\partial|\beta|^{2}}{\partial z^{i}}-\frac{d}{d s}\left(\frac{\partial|\beta|^{2}}{\partial \eta^{i}}\right) \frac{d s}{d t} \\
= & -\alpha^{2} b_{i} b_{\bar{l}} \frac{d^{2} \bar{z}^{l}}{d s^{2}}+\bar{\beta} F_{i l} \eta^{l}+\left(F_{i \bar{l}}-b_{i} q_{\bar{l}}\right) \bar{\eta}^{l} \\
= & -\alpha^{2} b_{i} b_{\bar{l}} \frac{d^{2} \bar{z}^{l}}{d s^{2}}+\bar{\beta} F_{i l} \eta^{l}+\left(b_{l} \frac{\partial b_{\bar{r}}}{\partial z^{i}}-b_{i} \frac{\partial b_{\bar{r}}}{\partial z^{l}}\right) \bar{\eta}^{r} \eta^{l}-\left(b_{\bar{l}} \frac{\partial b i}{\partial \bar{z}^{r}}+b_{i} \frac{\partial b_{\bar{l}}}{\partial \bar{z}^{r}}\right) \bar{\eta}^{r} \bar{\eta}^{l},
\end{aligned}
$$

where $\Gamma_{\bar{j} i}^{\bar{r}}:=\frac{1}{2} a^{\bar{r} k}\left\{\frac{\partial a_{k \bar{j}}}{\partial z^{i}}-\frac{\partial a_{i \bar{j}}}{\partial z^{k}}\right\}$ and $\Gamma_{\bar{j} \bar{l}}^{\bar{r}}:=\frac{1}{2} a^{\bar{r} k}\left\{\frac{\partial a_{k \bar{j}}}{\partial \bar{z}^{\bar{j}}}+\frac{\partial a_{k \bar{l}}}{\partial \bar{z}^{j}}\right\}$ are the coefficients of Levi-Civita connection of $a_{i \bar{j}} ; F_{i l}:=\frac{\partial b_{l}}{\partial z^{i}}-\frac{\partial b i}{\partial z^{l}}$ are complex electromagnetic tensor fields of the functions $\beta$.

Plugging the relation (4.6) into the formulas of $E_{i}\left(|\beta|^{2}\right)$, we obtain:

$$
\begin{aligned}
E_{i}\left(|\beta|^{2}\right)= & \frac{\bar{\beta}^{2} \alpha^{2}}{|\beta|^{2}} b_{i} b_{l} \frac{d^{2} z^{l}}{d s^{2}}+\left(\frac{\bar{\beta}^{2}}{|\beta|^{2}} b_{i} \frac{\partial b_{l}}{\partial z^{r}} \eta^{r}+\bar{\beta} F_{i l}+b_{l} \frac{\partial b_{\bar{r}}}{\partial z^{i}} \bar{\eta}^{r}\right) \eta^{l} \\
& +\left(\frac{\bar{\beta}^{2}}{|\beta|^{2}} b_{i} \frac{\partial b_{r}}{\partial \bar{z}^{l}} \eta^{r}-\bar{\beta} \frac{\partial b i}{\partial \bar{z}^{l}}\right) \bar{\eta}^{l} .
\end{aligned}
$$

Now, substituting above formulas of $E_{i}\left(\alpha^{2}\right)$ and $E_{i}\left(|\beta|^{2}\right)$ in (4.4), it results

$$
\begin{aligned}
& {\left[\alpha^{2}|\beta| a_{i \bar{l}} \frac{d^{2} \bar{z}^{l}}{d s^{2}}-\left(\frac{\alpha \bar{\beta}^{2}}{|\beta|^{2}} b_{i} \frac{\partial b_{r}}{\partial \bar{z}^{l}} \eta^{r}-\alpha \bar{\beta} \frac{\partial b i}{\partial \bar{z}^{l}}-|\beta| a_{i \bar{r}} \Gamma_{\bar{j}}^{\bar{r}} \bar{\eta} \bar{\eta}^{j}\right) \bar{\eta}^{l}\right] } \\
+ & {\left[\frac{\alpha^{3} \bar{\beta}^{2}}{|\beta|^{2}} b_{i} b_{l} \frac{d^{2} z^{l}}{d s^{2}}+\left(\frac{\alpha \bar{\beta}^{2}}{|\beta|^{2}} b_{i} \frac{\partial b_{l}}{\partial z^{r}} \eta^{r}+\alpha \bar{\beta} F_{i l}+\alpha b_{l} \frac{\partial b_{\bar{r}}}{\partial z^{i}} \bar{\eta}^{r}+2|\beta| a_{l \bar{r}} \Gamma_{\bar{j} i}^{\bar{r}} \bar{\eta}^{j}\right) \eta^{l}\right]=0 . }
\end{aligned}
$$

Further, following the same arguments as in [15, p. 93], the vanishing of the both brackets above:

$$
\begin{aligned}
& \frac{\alpha^{3} \bar{\beta}^{2}}{|\beta|^{2}} b_{i} b_{l} \frac{d^{2} z^{l}}{d s^{2}}+\left(\frac{\alpha \bar{\beta}^{2}}{|\beta|^{2}} b_{i} \frac{\partial b_{l}}{\partial z^{r}} \eta^{r}+\alpha \bar{\beta} F_{i l}+\alpha b_{l} \frac{\partial b_{\bar{r}}}{\partial z^{z}} \bar{\eta}^{r}+2|\beta| a_{l \bar{r}} \Gamma_{\frac{\bar{j}}{\bar{r}}}^{\bar{r}} \bar{\eta}^{j}\right) \eta^{l}=0 \\
& \alpha^{2}|\beta| a_{i \bar{l}} \frac{d^{2} \bar{z}^{l}}{d s^{2}}-\left(\frac{\alpha \bar{\beta}^{2}}{|\beta|^{2}} b_{i} \frac{\partial b_{r}}{\partial \bar{z}^{l}} \eta^{r}-\alpha \bar{\beta} \frac{\partial b i}{\partial \bar{z}^{l}}-|\beta| a_{i \bar{r}} \Gamma_{\bar{j} \overline{\bar{j}}}^{\overline{\bar{r}}} \bar{\eta}^{j}\right) \bar{\eta}^{l}=0 .
\end{aligned}
$$

are called the equations of a complex geodesic of Lorentz type.

By conjugation everywhere in the equations (4.8) and contracting then with $a^{\bar{i} h}$, it gives

$$
\alpha^{2}|\beta| \frac{d^{2} z^{h}}{d s^{2}}-\left(\frac{\alpha \beta^{2}}{|\beta|^{2}} b^{h} \frac{\partial b_{\bar{r}}}{\partial z^{l}} \bar{\eta}^{r}-\alpha \beta a^{\bar{\imath} h} \frac{\partial b \bar{l}}{\partial z^{l}}-|\beta| \Gamma_{j l}^{h} \eta^{j}\right) \eta^{l}=0 .
$$

Taking into account formulas (4.3), we obtain

$$
\frac{d^{2} z^{h}}{d s^{2}}+\Gamma_{j l}^{h} \frac{d z^{j}}{d s} \frac{d z^{l}}{d s}+\left(\frac{\beta}{|\beta|} a^{\bar{\imath} h} \frac{\partial b \bar{\imath}}{\partial z^{l}}-\frac{\beta^{2}}{|\beta|^{3}} b^{h} \frac{\partial b_{\bar{r}}}{\partial z^{l}} \bar{\eta}^{r}\right) \frac{d z^{l}}{d s}=0 .
$$


We note that (4.10) can be rewritten in the form $\frac{d^{2} z^{h}}{d t^{2}}+2 G^{h}(z(t), \eta(t))=0$, where $G^{h}:=\frac{1}{2} \Gamma_{j l}^{h} \eta^{j} \eta^{l}+\frac{\alpha}{2}\left(\frac{\beta}{|\beta|} a^{\bar{\imath} h} \frac{\partial b \bar{\imath}}{\partial z^{l}}-\frac{\beta^{2}}{|\beta|^{3}} b^{h} \frac{\partial b_{\bar{r}}}{\partial z^{l}} \bar{\eta}^{r}\right) \eta^{l}$. Using the changes of complex coordinates on $T^{\prime} M$, by direct computation we can prove that the functions $G^{h}$ are the coefficients of a complex spray on $T^{\prime} M$. Now, recall from [15] that a complex spray determines a (c.n.c) $\stackrel{c}{N}$ by the rule $\stackrel{c}{N_{j}^{i}}=\frac{\partial G^{i}}{\partial \eta^{j}}$ which, following the real case, will be called of Cartan type.

On the other hand, any (c.n.c.) $N$, by contraction determines a complex spray, i.e., $N_{j}^{i} \eta^{j}=2 G^{i}$. Paying more attention to the spray resulted from (4.10) we observe that it is contracted with $\eta^{l}$. Therefore, (4.10) leads to:

Proposition 4.3. The functions

$$
\stackrel{L o}{N_{l}^{h}}(z, \eta):=N_{l}^{h}+\frac{\alpha \beta}{|\beta|} a^{\bar{\imath} h} \frac{\partial b \bar{\imath}}{\partial z^{l}}-\frac{\alpha \beta^{2}}{|\beta|^{3}} b^{h} \frac{\partial b_{\bar{r}}}{\partial z^{l}} \bar{\eta}^{r}
$$

are the coefficients of a (c.n.c.).

We called this (c.n.c.) of Lorentz type.

It is obvious that $\stackrel{c}{N}_{j}^{i}(z, \eta)=\frac{1}{2} \frac{\partial\left(N_{k}^{i} \eta^{k}\right)}{\partial \eta^{j}}$ and both (c.n.c.) are inhomogeneous with respect to $\eta$.

By direct computation we can find the link between Chern-Finsler (c.n.c)

Lo and $N_{k}^{i}$ (c.n.c.) :

$$
\stackrel{C F}{N_{k}^{i}}=N_{k}^{i}+\frac{1}{\gamma} \varepsilon^{i}\left(\beta^{2} \frac{\alpha|| b||^{2}+|\beta|}{|\beta|^{3}} \frac{\partial b_{\bar{m}}}{\partial z^{k}} \bar{\eta}^{m}+\frac{\partial b_{l}}{\partial z^{k}} \eta^{l}-b^{\bar{m}} \frac{\partial a_{l \bar{m}}}{\partial z^{k}} \eta^{l}-\frac{\alpha \beta}{|\beta|} b^{\bar{m}} \frac{\partial b_{\bar{m}}}{\partial z^{k}}\right) .
$$

Note that as yet we have not reached to the first set (4.7) of equations. In [15], for the general framework of complex Lagrange spaces, it is proved that it is related to the weakly Kähler condition of the Chern-Finsler connection. We think that it is natural to view what consequences brings the existence of a complex geodesic of Lorentz type. Therefore, contracting the relation (4.7) by $\eta^{i}$ and taking into account that $F_{i l} \eta^{i} \eta^{l}=0 ; a_{l \bar{r}} \Gamma_{\bar{j} i}^{\bar{r}} \bar{\eta}^{j} \eta^{i}=0$, we find

$$
\alpha^{2} \bar{\beta} b_{l} \frac{d^{2} z^{l}}{d s^{2}}+\left(\bar{\beta} \frac{\partial b_{r}}{\partial z^{l}} \eta^{r}+\beta \frac{\partial b_{\bar{r}}}{\partial z^{l}} \bar{\eta}^{r}\right) \eta^{l}=0
$$

Replacing (4.13) in (4.9) multiplied with $\frac{\bar{\beta} b_{l}}{|\beta|}$ and respectively (4.13) multiplied with $\frac{\alpha \bar{\beta} b_{i}}{\left|\beta^{2}\right|}$ in (4.7), it is obtained

$$
\begin{gathered}
\beta \frac{\alpha|| b||^{2}+|\beta|}{|\beta|} \frac{\partial b_{\bar{m}}}{\partial z^{r}} \bar{\eta}^{m} \eta^{r}+\bar{\beta}\left(\frac{\partial b_{r}}{\partial z^{l}}-b^{\bar{m}} \frac{\partial a_{l \bar{m}}}{\partial z^{r}}\right) \eta^{l} \eta^{r}-\alpha|\beta| b^{\bar{m}} \frac{\partial b_{\bar{m}}}{\partial z^{r}} \eta^{r}=0 \\
\left(\alpha \bar{\beta} F_{i l}+\alpha b_{l} \frac{\partial b_{\bar{r}}}{\partial z^{i}} \bar{\eta}^{r}+2|\beta| a_{l \bar{r}} \Gamma_{\bar{j} i}^{\bar{r}} \bar{\eta}^{j}\right) \eta^{l}=\alpha b_{i} \frac{\partial b_{\bar{m}}}{\partial z^{r}} \bar{\eta}^{m} \eta^{r}
\end{gathered}
$$


Furthermore, we can obtain a significant consequence of the equations (4.7) and (4.8). Namely, corroborating the relations (4.14) and (4.15) with the weakly Kähler condition, it results

Proposition 4.4. A complex Randers space $(M, \alpha+|\beta|)$ for which there exists a complex geodesic of Lorentz type is weakly Kähler.

Finally, taking into account (4.12) and (4.14) we obtain

Colorallary 4.1. If there exists a complex geodesic of Lorentz type, then $N_{k}^{i}$ $C F$

and $N_{k}^{i}$ determine the same spray.

In this note we made an approach for the main tools in the geometry of complex Randers spaces. The matter is far from being exhausted. It was not our goal here to study complex Randers spaces with constant holomorphic curvature or the Zermelo navigation problem ([8]), to give some examples. It is hoped that the complex Randers spaces will enrich the complex Finsler geometry, bringing forward new interesting questions. It is not lost of interest the study of complex Kropina spaces, or a general study for complex $(\alpha, \beta)$ metrics.

\section{References}

[1] M. Abate and G. Patrizio, Finsler Metrics-A Global Approach. With Applications to Geometric Function Theory, Lecture Notes in Mathematics, 1591. Springer-Verlag, Berlin, 1994, x+180 pp.

[2] T. Aikou, Projective Flatness of Complex Finsler Metrics, Publ. Math. Debrecen 63 (2003), no. 3, 343-362.

[3] N. Aldea, Complex Finsler spaces of constant holomorphic curvature, Differential geometry and its applications, 179-190, Matfyzpress, Prague, 2005.

[4] N. Aldea and G. Munteanu, $(\alpha, \beta)$ - complex Finsler metrics, Proceedings of the 4th International Colloquium "Mathematics in Engineering and Numerical Physics", 1-6, BGS Proc., 14, Geom. Balkan Press, Bucharest, 2007.

[5] _ On the geometry of complex Randers spaces, Proc. of the 14-th Nat. Sem. on Finsler, Lagrange and Hamilton spaces, Braşov, 2006, 1-8.

[6] D. Bao, S. S. Chern, and Z. Shen, An introduction to Riemann-Finsler geometry, Graduate Texts in Mathematics 200, Springer-Verlag, New York, 2000, xx+431 pp.

[7] D. Bao and C. Robles, On Randers spaces of constant flag curvature, Rep. Math. Phys. 51 (2003), no. 1, 9-42.

[8] D. Bao, C. Robles, and Z. Shen, Zermelo navigation on Riemannian manifolds, J. Differential Geom. 66 (2004), no. 3, 377-435.

[9] B. Chen and Y. Shen, Complex Randers Metrics, communicated to Conference Zhejiang Univ., China, July, 2007.

[10] M. Fukui, Complex Finsler manifolds, J. Math. Kyoto Univ. 29 (1989), no. 4, 609-624.

[11] R. S. Ingarden, On the geometrically absolute optical representation in the electron microscope, Trav. Soc. Sci. Lett. Wrochlaw, Ser. B. 1957 (1957), no. 3, 60 pp.

[12] S. Kobayashi and C. Horst, Topics in complex differential geometry. Complex Differential Geometry, 4-66, DMV Sem. 3. Birkhäuser, Basel, 1983.

[13] M. Matsumoto, Randers spaces of constant curvature, Rep. Math. Phys. 28 (1989), no. $2,249-261$. 
[14] R. Miron, The geometry of Ingarden spaces, Rep. Math. Phys. 54 (2004), no. 2, 131-147.

[15] G. Munteanu, Complex Spaces in Finsler, Lagrange and Hamilton Geometries, Fundamental Theories of Physics, 141. Kluwer Academic Publishers, Dordrecht, 2004, xii+221 pp.

[16] S. Nishikawa, Harmonic maps of Finsler manifolds, Topics in differential geometry, Ed. Academiei Române, Bucureşti 2008, 208-247.

[17] H. L. Royden, Complex Finsler metrics, Complex differential geometry and nonlinear differential equations (Brunswick, Maine, 1984), 119-124, Contemp. Math., 49, Amer. Math. Soc., Providence, RI, 1986.

[18] A. Spiro, The Structure Equations of a Complex Finsler Manifold, Asian J. Math. 5 (2001), no. 2, 291-326.

[19] P.-M. Wong, A survey of complex Finsler geometry. Finsler geometry, Sapporo 2005-in memory of Makoto Matsumoto, 375-433, Adv. Stud. Pure Math. 48, Math. Soc. Japan, Tokyo, 2007.

[20] R. Yan, Connections on complex Finsler manifold, Acta Math. Appl. Sin., Engl. Ser. 19, (2003), no. 3, 431-436.

[21] H. Yasuda and H. Shimada, On Randers spaces of scalar curvature, Rep. Mathematical Phys. 11 (1977), no. 3, 347-360.

Nicoleta Aldea

FACUlty OF Mathematics AND Informatics

TRANSILVANIA UNIVERSITY

IUliu Maniu 50, Braşov 500091, Romania

E-mail address: nicoleta.aldea@lycos.com

Gheorghe Munteanu

FACULTY OF MATHEMATICS AND INFORMATICS

TRANSILVANIA UNIVERSITY

Iuliu Maniu 50, Braşov 500091, Romania

E-mail address: gh.munteanu@unitbv.ro 